\title{
An immunoperoxidase study of epithelial marker antigens in ulcerative colitis with dysplasia and carcinoma
}

\author{
DC ALLEN, JD BIGGART, JC ORCHIN, HEATHER FOSTER
}

From the Histopathology Laboratory, Belfast City Hospital, Belfast

SUMMARY An immunoperoxidase technique was applied to formalin and Helly fixed paraffin wax sections from cases of ulcerative colitis complicated by dysplasia and carcinoma for carcinoembryonic antigen and components of the colonic secretory immunoglobulin system-namely, secretory component, $\operatorname{Ig} A$, and $J$ chain. Sections from both resection specimens and mucosal biopsies were available. Intensity of immunostaining was assessed qualitatively. There was appreciable variation in expression of carcinoembryonic antigen and secretory component antigens. Carcinoembryonic antigen stained heavily in dysplasia and carcinoma while these tissues showed only focal light staining for secretory component. Normal tissue stained heavily for secretory component. The variation in staining intensity for both carcinoembryonic antigen and secretory component in inflamed and regenerative mucosa precluded their use as a reliable diagnostic aid in discriminating these tissues from true dysplasia. Loss of secretory component production or transport or both may be incurred during malignant change, but it should not be assessed as an isolated index of epithelial maturity. The relation with mucosal plasma cells warrants further study to determine more fully the factors affecting tissue secretory component expression.

Since the initial report in $1925^{1}$ carcinoma has been recognised as an uncommon but important complication in ulcerative colitis. Warren and Sommers ${ }^{2}$ postulated a precancerous mucosal change and later, in 1967, Morson and Pang ${ }^{3}$ established the use of rectal biopsy as an aid to the early diagnosis of cancer in ulcerative colitis. The subsequent provision of both multiple rectal and colonic biopsies has led to problems of histological interpretation as actively regenerating colitic mucosa may closely resemble dysplasia. $^{45} \mathrm{~A}$ recent international classification of the mucosal changes seen in ulcerative colitis will prove useful in assessing the risk of malignancy. ${ }^{6}$ Several additional techniques are being evaluated as diagnostic aids with varying results. These include mucin histochemistry, ${ }^{78}$ epithelial cell kinetics, ${ }^{9}$ and flow cytometry. ${ }^{10}$ Immunohistochemical studies have illustrated the use of tumour associated antigens, such as carcinoembryonic antigen, ${ }^{11}$ as a tissue marker for potential and established malignancy. A different approach has been to show the loss of normal cellular function

Accepted for publication 18 September 1984 incurred during the evolution of malignancy. One such function is the production and secretion of $\operatorname{IgA}$ ? by the gut mucosa. Plasma cells in the lamina propria secrete $\operatorname{IgA}$ as a dimer linked by a joining $(\mathrm{J}) \stackrel{\text {. }}{\text {. }}$ chain. Dimeric IgA is then attached to a polypeptide, secretory component (SC), manufactured in $₹$ the mucosal epithelium. The assembled $\operatorname{IgA} \mathrm{A}-\mathrm{J}-\mathrm{SC}$ 음 unit is then transported through the epithelium to $D$ the gut lumen. ${ }^{12}$ This study concerns the immunohistochemical demonstration of carcinoembryonic N antigen, secretory component, $\operatorname{IgA}$, and $\mathrm{J}$ chain in benign and malignant mucosal changes of ulcerative colitis.

\section{Material and methods}

The material included in this study came from seven $\stackrel{\mathbb{\complement}}{+}$ patients with ulcerative colitis who underwent total 0

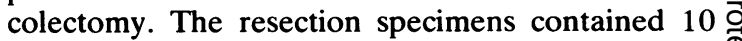
primary carcinomas. A total of 23 mucosal biopsies $\stackrel{\mathbb{Q}}{\Omega}$ (6 rectal, 17 colonic) all taken less than 12 months $\mathbb{\perp}$ before resection were available for examination. The resection specimens were opened at the time of 
Details of seven patients and 10 primary cancers arising as a complication of ulcerative colitis included in this study

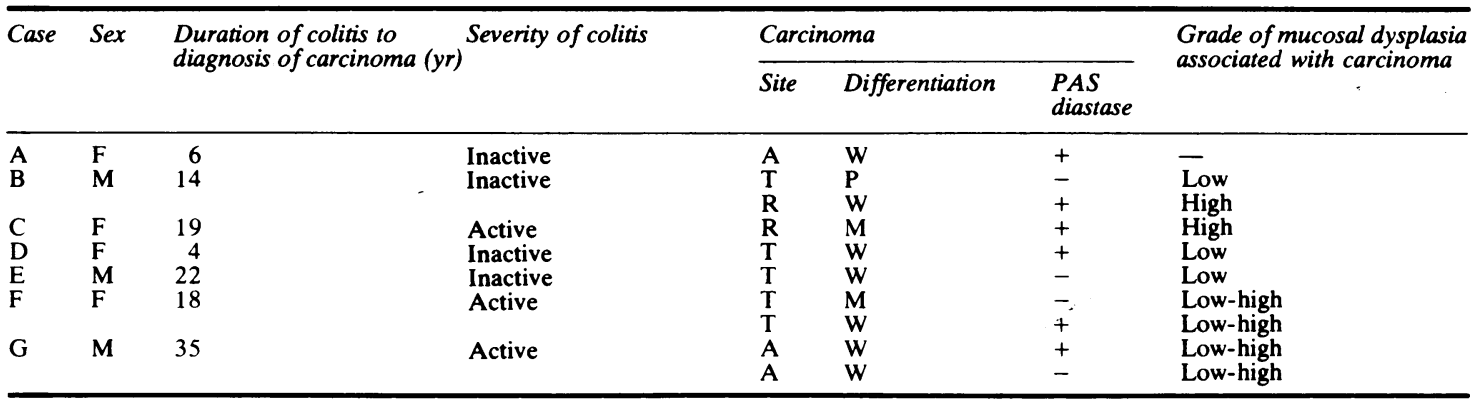

$\mathrm{A}=$ ascending colon; $\mathbf{T}=$ transverse colon; $\mathbf{R}=$ rectum.

Differentiation: $\mathrm{W}=$ well; $\mathrm{M}=$ moderate; $\mathrm{P}=$ poor

PAS = periodic acid Schiff.

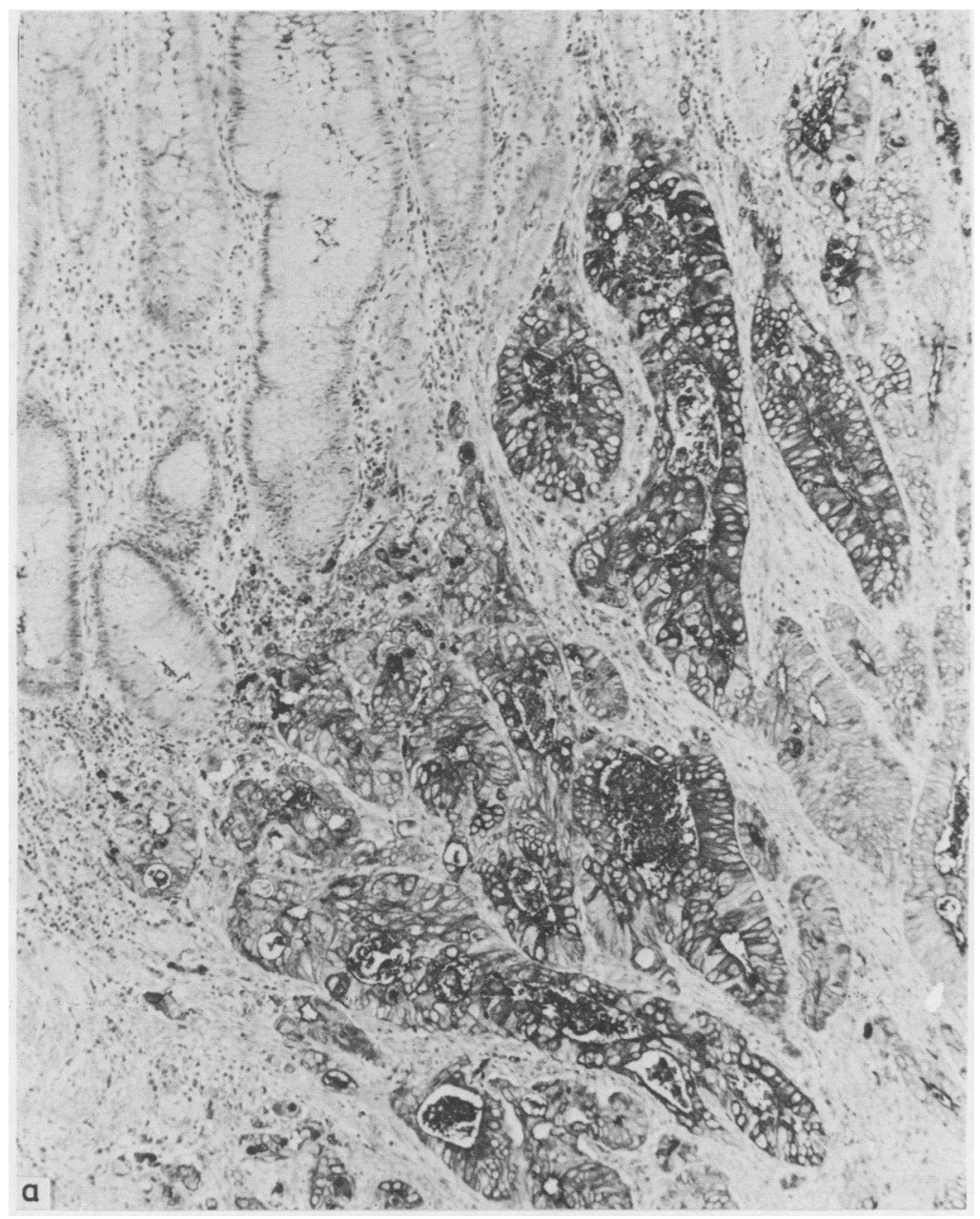

Fig. 1 Invasive adenocarcinoma with normal adjacent colonic mucosa.

(a) Carcinoembryonic antigen. Apical cytoplasmic staining with lumenal rim and central debris; confined to the carcinoma. Immunoperoxidase. $\times 100$. 


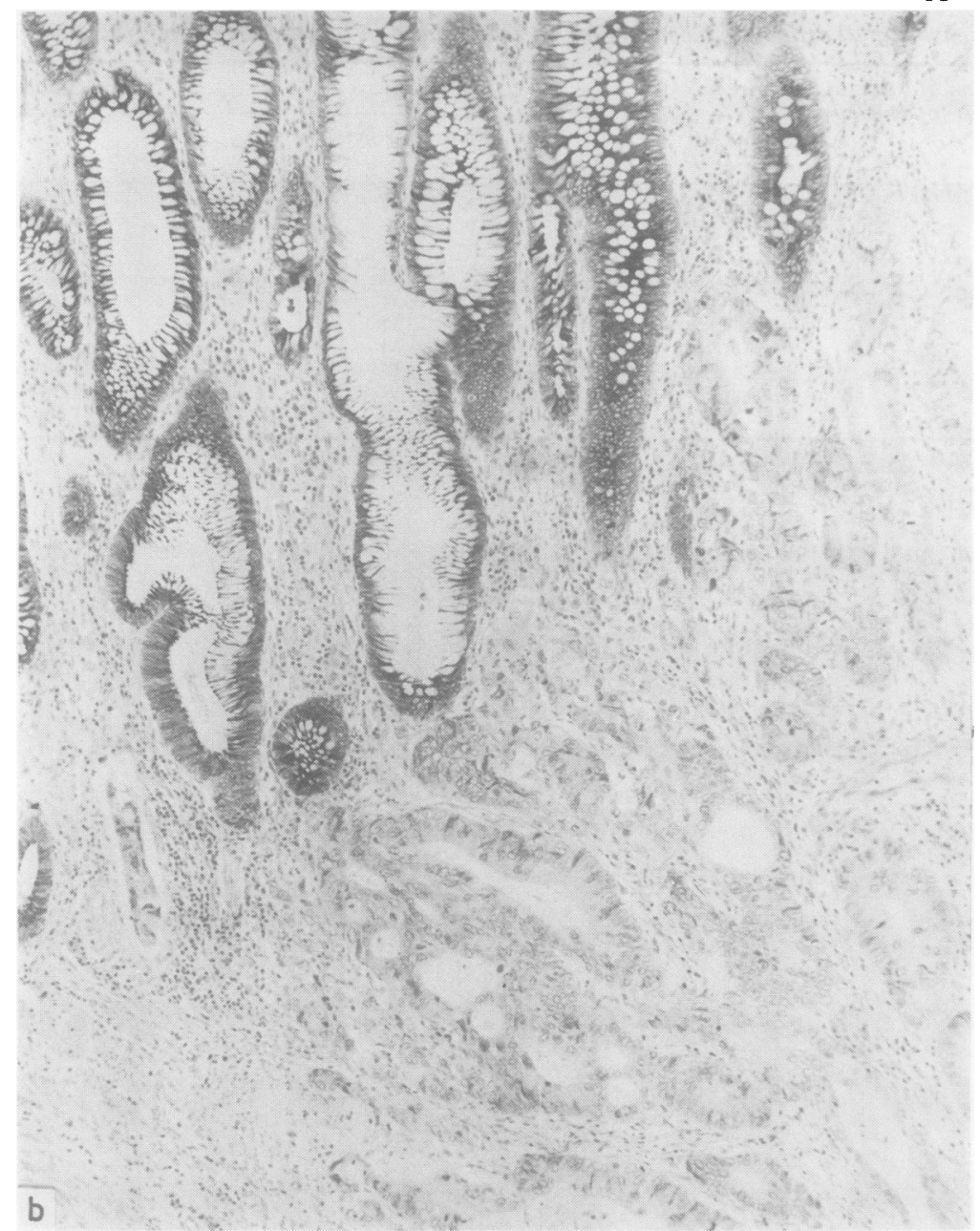

Fig. 1(b) Secretory component. Normal mucosa shows diffuse cytoplasmic staining in non-mucin secreting columnar cells. Tumour negative. Immunoperoxidase. $\times 100$.

operation and all tissues were immediately fixed in $4 \%$ aqueous formaldehyde. Tissues were secondarily fixed in Helly's fluid and processed to paraffin wax embedding. Biopsies were examined through multiple levels, and an average of five blocks were selected from each resection for inclusion in this study. The mucosa was assessed on haematoxylin and eosin appearances for dysplasia according to a standardised international classification. ${ }^{6}$ Tumours were graded as poorly, moderately, or well differentiated. The main clinicopathological features of the cases are summarised in the Table. A more detailed discussion of this material forms the basis of another report ( $p$ 30).

Adjacent sections of biopsy and resection tissues were stained for carcinoembryonic antigen and secretory component using an unlabelled antibody peroxidase-antiperoxidase technique. Sections from selected blocks were stained for $\operatorname{IgA}$ and $J$ chain. Deparaffinised, rehydrated sections were treated with hydrogen peroxide to block endogenous peroxidase activity and reduce both non-specific background staining and non-specific cross reaction between carcinoembryonic antigen antibody and related glycoproteins. ${ }^{13}$ Both non-digested and trypsinised sections were prepared. Primary antibodies (rabbit), bridging antibody (swine), and peroxidase-antiperoxidase (rabbit) were obtained commercially (Dakopatts). The secretory component antibody reacted strongly on immunoelectrophoresis with human secretory IgA (and free secretory component). The usual dilutions of rabbit anti-carcinoembryonic antigen and anti-secretory component were $1 / 3200$ and $1 / 200$ respectively. 


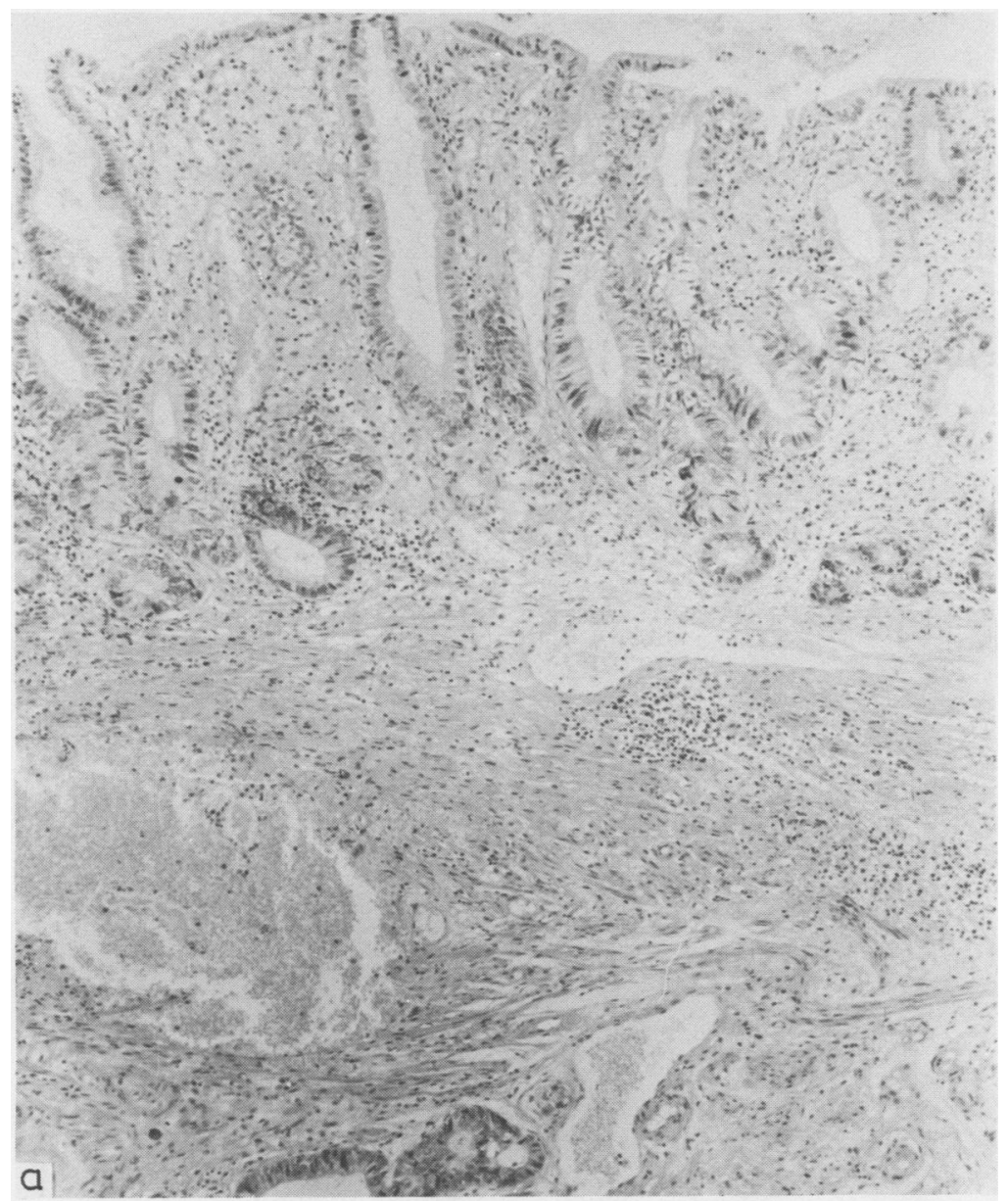

Fig. 2(a) Flat mucosal dysplasia with invasive adenocarcinoma in the submucosal tissues. Haematoxylin and eosin $\times 100$.

Each case was titred using doubling dilutions, however, and optimal titres varied between $1 / 1600$ and $1 / 6400$ and between $1 / 100$ and $1 / 800$ for carcinoembryonic antigen and secretory component respectively. The bridging antibody and rabbit peroxidase-antiperoxidase were applied in dilutions of $1 / 50$ and $1 / 200$. All incubations were at $37^{\circ} \mathrm{C}$ for $30 \mathrm{~min}$. Colorisation was obtained using hydrogen peroxide and a diaminobenzidine substrate with a light haematoxylin counterstain. Immunoperoxidase staining was graded qualitatively as light, moderate, or heavy. Tissues were assessed by two of us (DCA, JDB).

Positive controls for carcinoembryonic antigen and secretory component were obtained using non- colitic colorectal carcinomas and normal colonic mucosa. Negative controls entailed omission in turn of primary and bridging antibodies, use of inappropriate antiserum and non-immune rabbit immunoglobulin, and application of hydrogen peroxide diaminobenzidine alone.

\section{Results}

\section{STAINING CHARACTERISTICS}

The principles and staining characteristics of carcinoembryonic antigen and secretory component are discussed with reference to Fig. 1(a) and (b) respectively. Carcinoembryonic antigen showed mild cytoplasmic staining particularly in the apex of the cell 


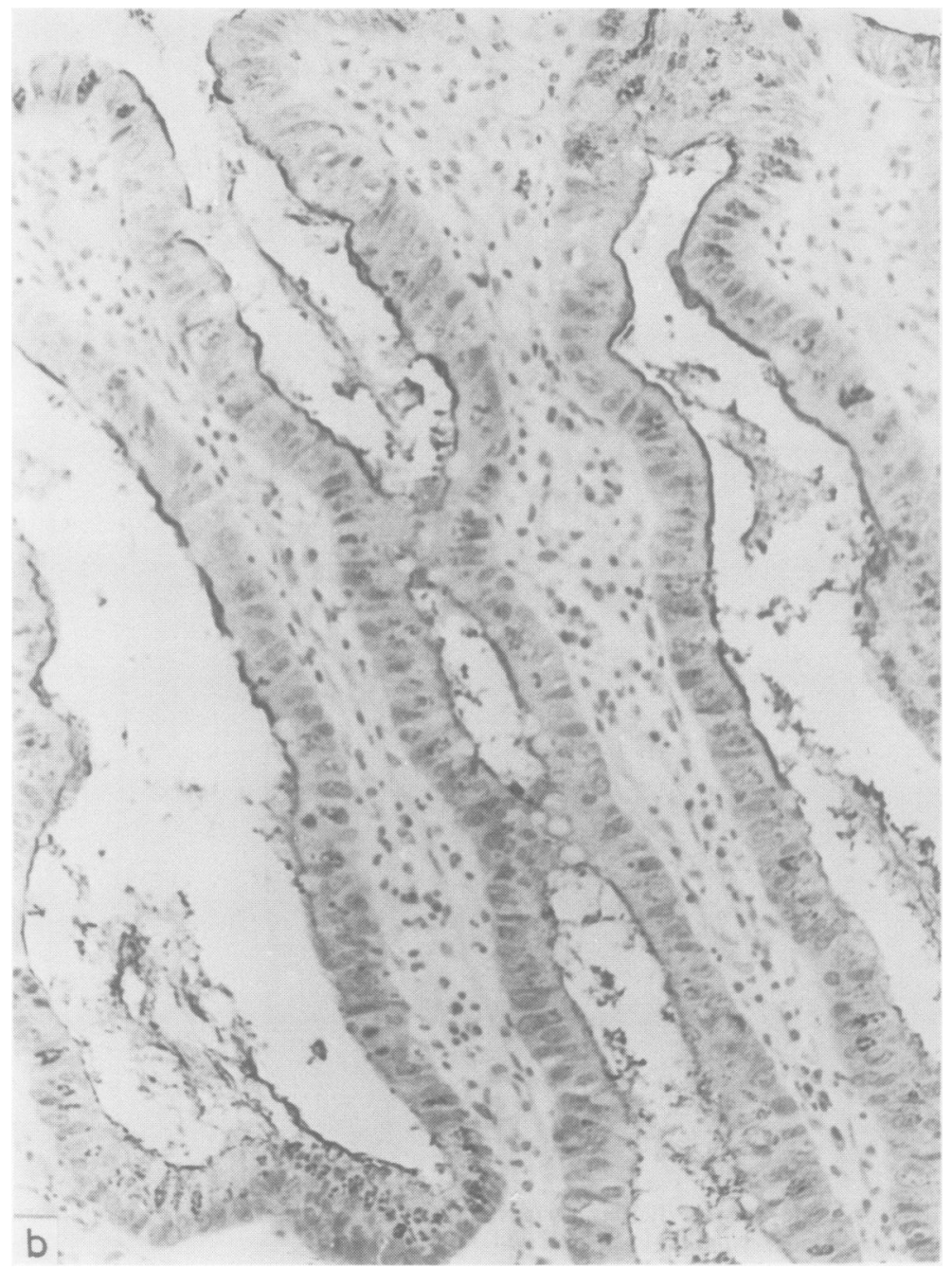

Fig. 2(b) High power view of the dysplastic mucosa stained for carcinoembryonic antigen. Note the fine, dark lumenal rim orientated to the epithelium.

Immunoperoxidase $\times 240$.

and occasionally in intercellular spaces. The emphasis of carcinoembryonic antigen staining was on a thin, irregular line corresponding to the glycocalyceal border occurring either as a glandular lumenal rim or over the surface epithelium. Often, tumour acini contained carcinoembryonic antigen positive debris and isolated tumour cells could be identified surrounded by a halo of carcinoembryonic antigen staining. Paneth cells showed a variable amount of light staining and goblet cells were negative. Primary antibody was titred to reduce any positive staining in normal mucosa but this did not eliminate absorption of staining from adjacent carcinoembryonic antigen positive mucosa. When endogenous activity was blocked, ${ }^{13}$ white blood cells and red blood cells did not show any appreciable staining.

Secretory component was positive in the non- 


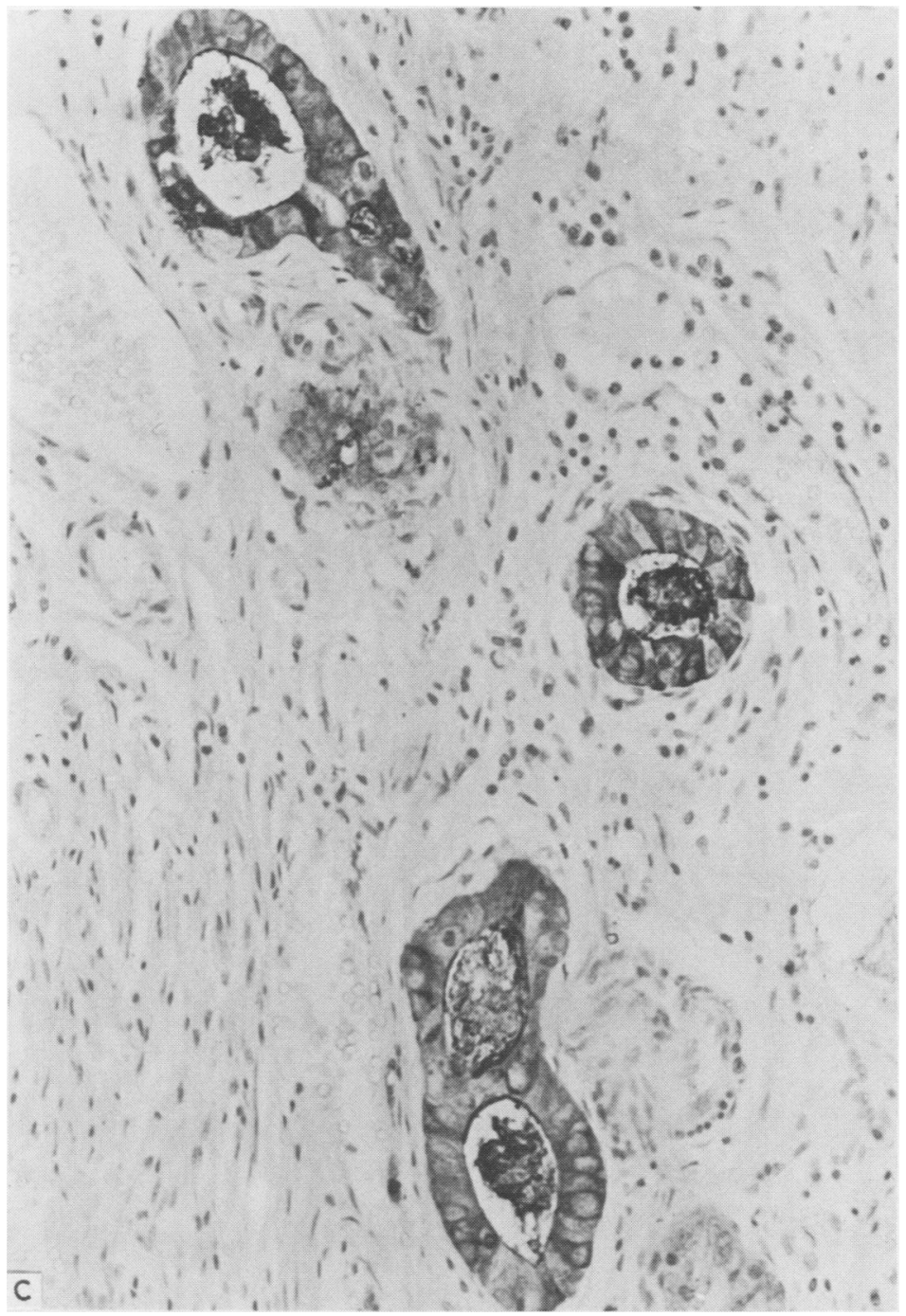

Fig. 2(c) Carcinoembryonic antigen. Foci of carcinoma seen in (a). Cytoplasmic and lumenal staining are present. Immunoperoxidase $\times 240$.

mucin secreting columnar epithelium of normal mucosa. Cytoplasmic staining was diffuse and lacked the apical and lumenal accentuation seen with carcinoembryonic antigen. Goblet and Paneth cells were negative. $\operatorname{IgA}$ and $\mathrm{J}$ chain diffusely stained the cytoplasm of plasma cells in the mucosal lamina propria and tumour stroma.

\section{TUMOUR}

Carcinoembryonic antigen was invariably present in areas of carcinoma; however, intensity of staining varied within some tumours and was uniform in others. Staining intensity could not be related to the degree of differentiation either within a given tumour or between tumours. The tumour growth pattern (ulcerated, villous, polypoid) did not dictate staining intensity, and deep invasive foci and metastatic tumour stained similarly to the superficial aspects of the primary tumour. Secretory component was negative in five cancers and positive in five (three with only sparse focal staining), all of which were well differentiated and four of which were mucin secreting. Tumour stromal $\operatorname{IgA}$ and $J$ chain were present in decreased amounts compared with 


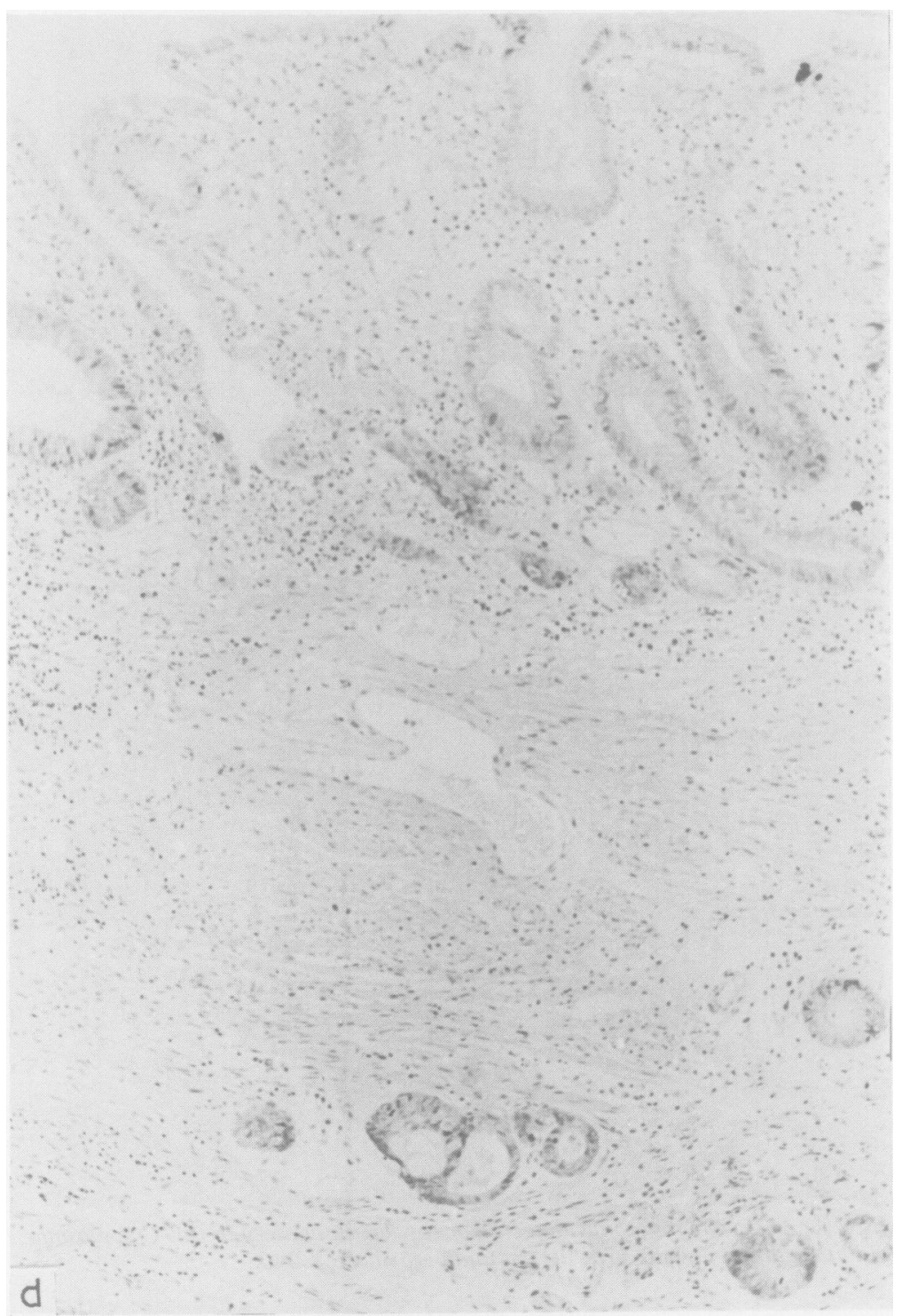

Fig. 2(d) Secretory component. Negative in both the dysplastic mucosa and carcinoma. Immunoperoxidase. $\times 100$.

adjacent mucosa but were present in three tumours that did not show secretory component staining.

REGENERATIVE AND DYSPLASTIC MUCOSA

In general, carcinoembryonic antigen was positive in dysplastic mucosa, whereas secretory component was negative (Fig. 2). This principle applied to mucosa in which there was no doubt on routine histological assessment about the presence of dysplasia. Occasionally, dysplastic mucosa showed focal light staining for secretory component, especially in polypoid adenomatous foci. The intensity of $\stackrel{\varrho}{\mathscr{C}}$ carcinoembryonic antigen in dysplastic mucosa was $\mathbb{D}$ similar to that seen in carcinoma, and it correlated with glandular crypt to surface maturation when present. Interestingly, in some areas of villous mucosal dysplasia with apparent crypt to surface maturation the surface mucosal fronds showed positive carcinoembryonic antigen staining and were negative for secretory component, which implies a 


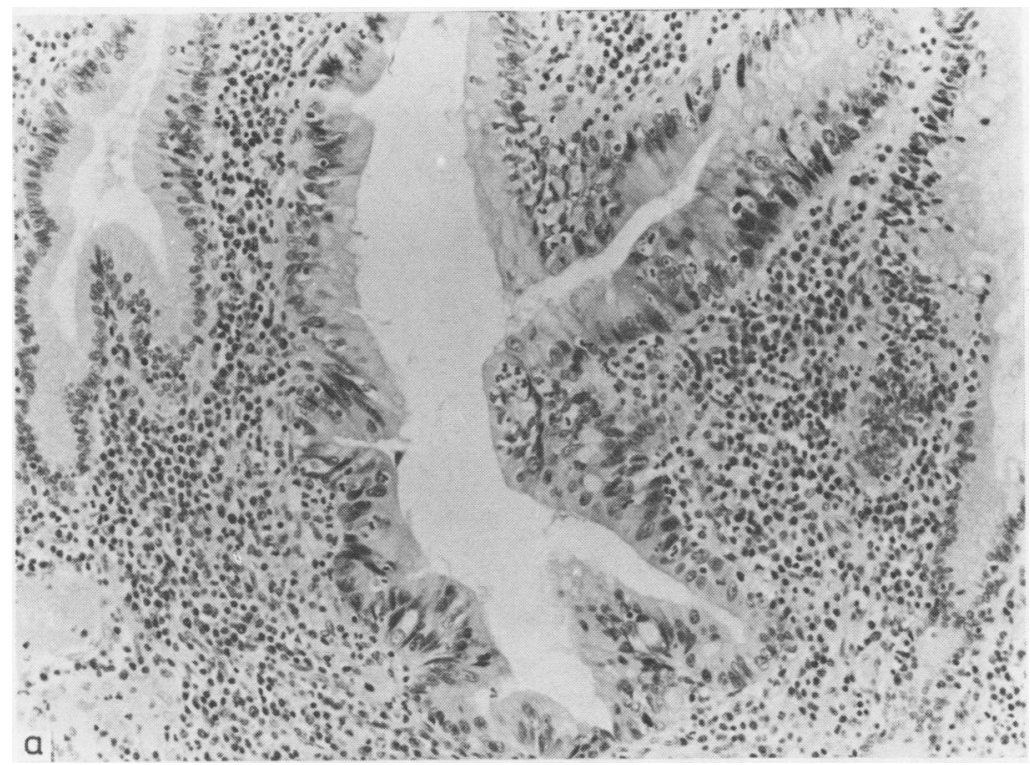

Fig. 3(a) Inflamed, regenerative mucosa. Negative for dysplasia. Note the two normal flanking glands. Haematoxylin and eosin. Original magnification $\times 250$. (b) An adjacent section stained for carcinoembryonic antigen (CEA). Positive cytoplasm and a fine lumenal rim in the regenerative gland. Compare with the two normal adjacent glands, negative for CEA. Immunoperoxidase. Original magnification $\times$ 250.

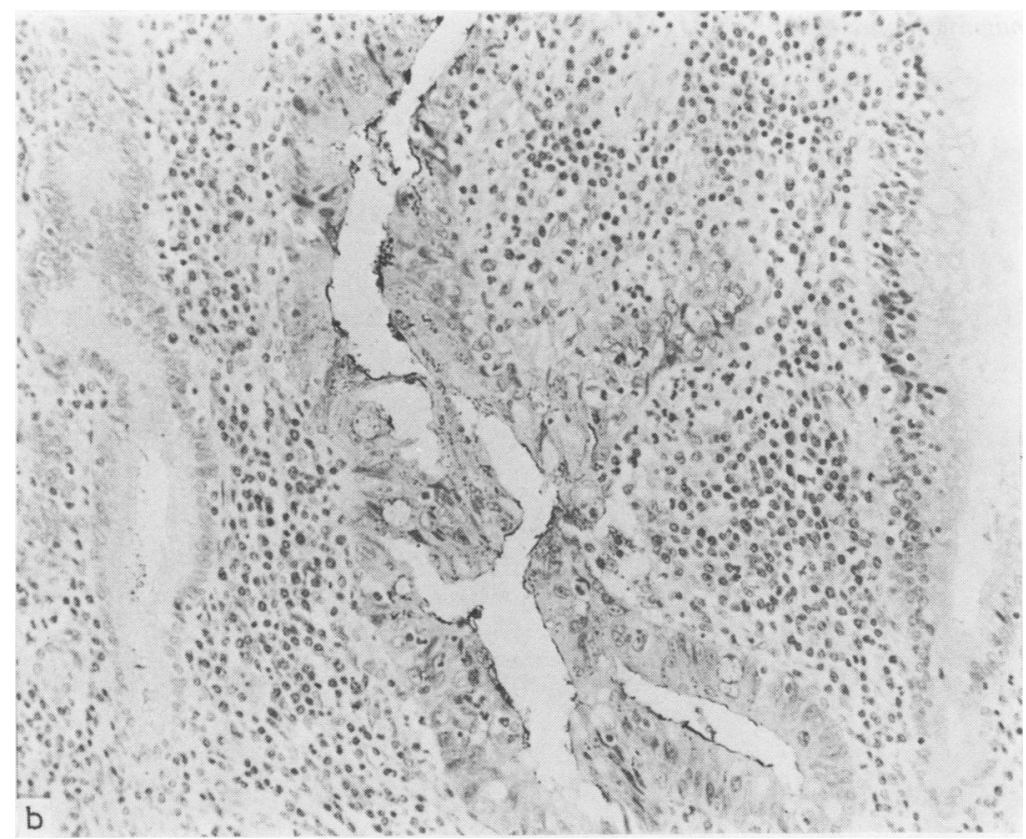




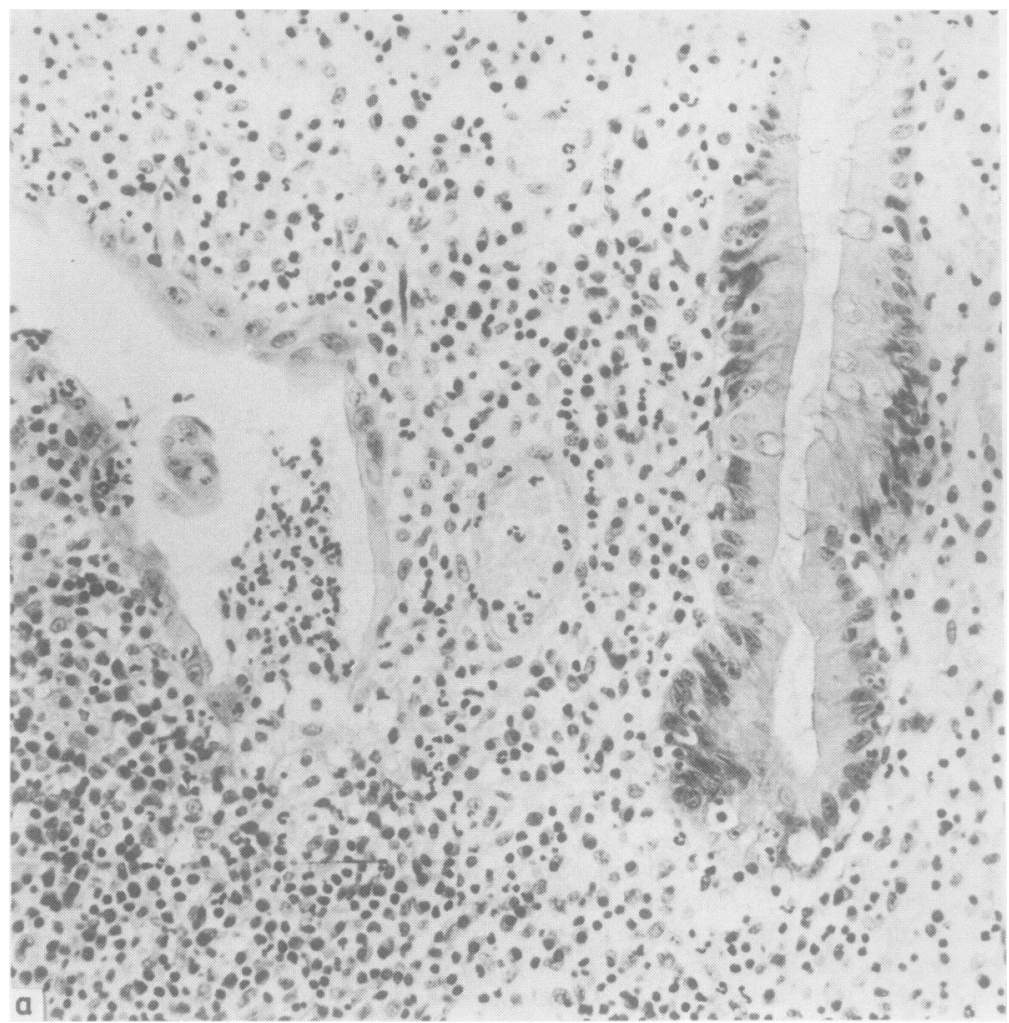

Fig. 4(a) Inflamed and regenerative mucosa. Haematoxylin and eosin. Original magnification $\times 260$. (b) An adjacent section stained for secretory component. The heavily inflamed gland shows loss of epithelial staining. Immunoperoxidase. Original magnification $\times 360$.

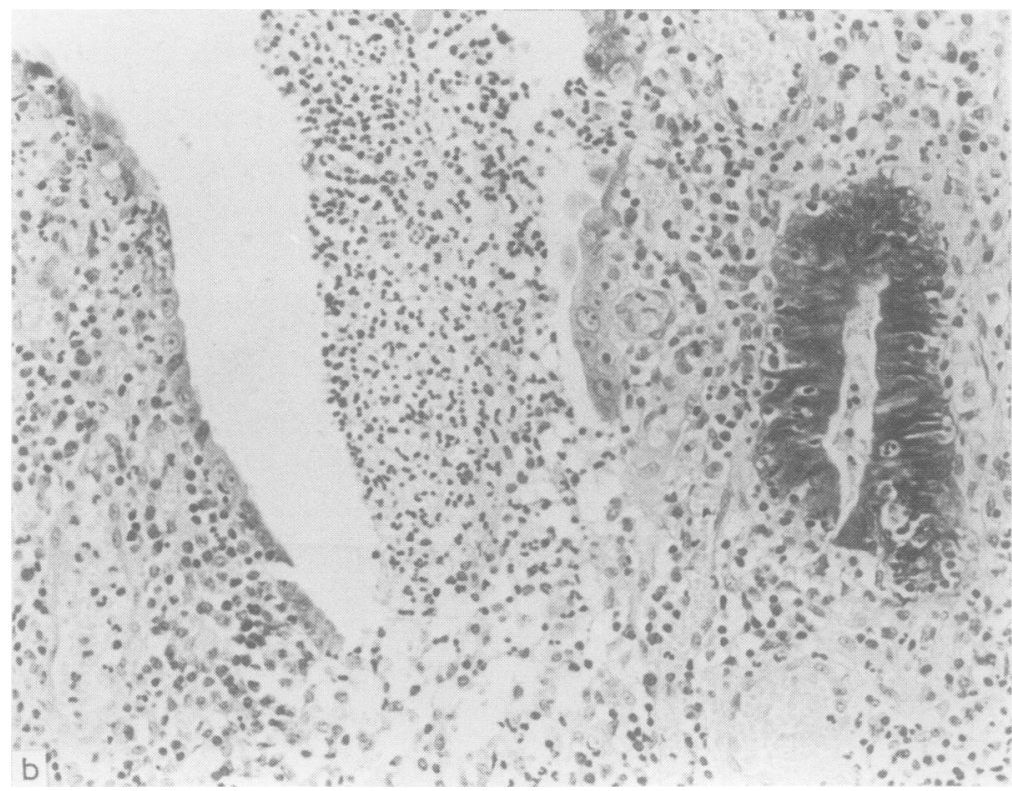




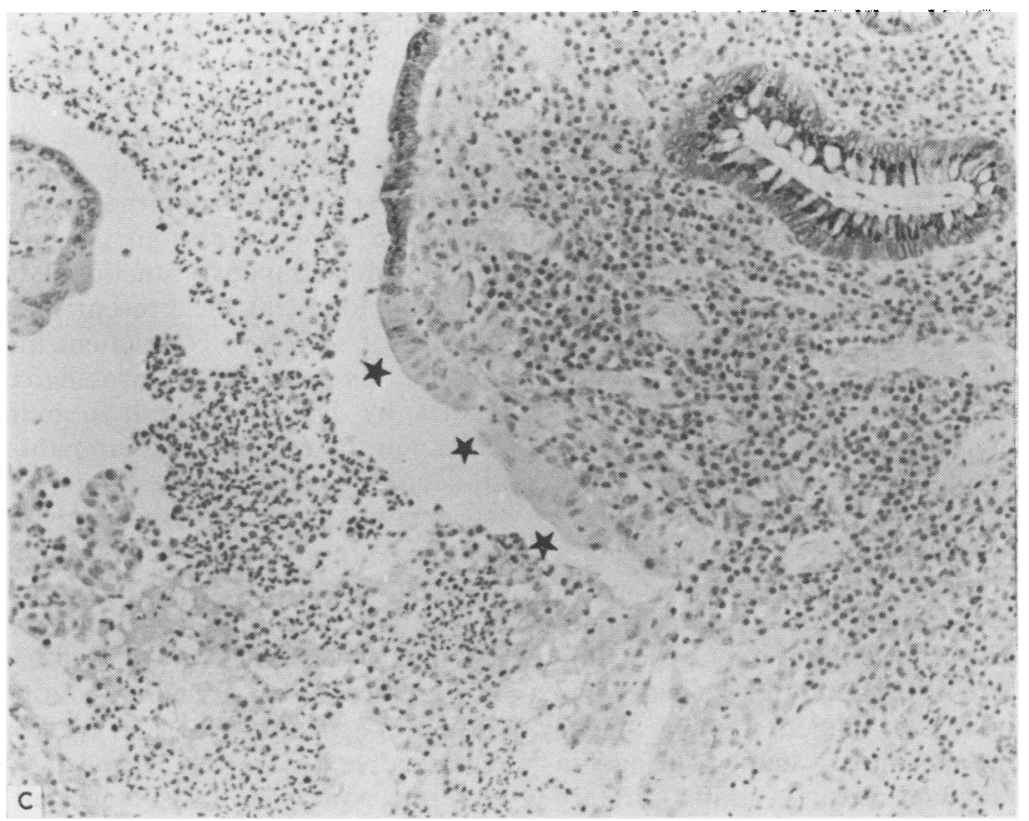

Fig. 4(c) A different case, again showing inflamed and regenerative epithelium with focal loss of secretory component. (asterisks). Immunoperoxidase. Original magnification $\times 260$.

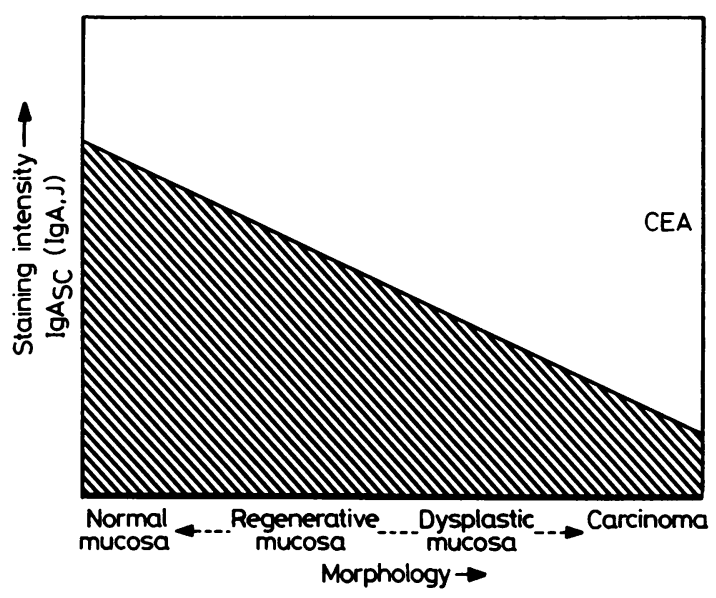

Fig. 5 Proposed model of immunostaining in ulcerative colitis

possible functional immaturity. Secretory component also showed variation in glandular staining sparing dysplastic crypts. Visual assessment of immunostaining was not a sensitive enough parameter to differentiate between low and high grades of dysplasia.
Mucosa with heavy inflammation and that assessed on histological examination as indefinite for dysplasia showed a wide range of staining with both carcinoembryonic antigen and secretory component. Inflamed mucosa with regenerative epithelium showed light to heavy carcinoembryonic antigen staining (Fig. 3), and, although usually stained at least moderately well for secretory component, did show multiple areas with loss of epithelial secretory component (Fig. 4).

The lamina propria content of $\operatorname{IgA}$ and $J$ chain related to the degree of inflammation irrespective of the epithelium being regenerative or dysplastic.

A model of immunostaining in ulcerative colitis is proposed (Fig. 5).

\section{Discussion}

The purpose of this study was to assess the use of immunostaining for carcinoembryonic antigen and secretory component as an aid to differentiating between regenerative hyperplasia and dysplasia in ulcerative colitis. These tissue marker antigens were evaluated with reference to routine morphology individually and in combination by use of paired sections.

In 1965 Gold and Freedman ${ }^{14}$ showed tumour 
specific antigens in human colonic carcinoma, and initial reports suggested that application of carcinoembryonic antigen immunostaining in tissue sections was a reliable indicator of malignant colorectal change. ${ }^{15}$ It was thought to be of potential use in discriminating between regenerative hyperplasia and dysplasia in colitic mucosa. " Subsequently, carcinoembryonic antigen has emerged as being less specific than originally thought, showing cross reactive staining with other normal bowel constituents ${ }^{1316}$ and carcinoembryonic antigen related antigens. ${ }^{17}$ It has been shown in lung tumours, ${ }^{17}$ normal and malignant gall bladder epithelium, ${ }^{18}$ and uterine cervical neoplasia. ${ }^{19}$ Several studies have noted its presence in normal ${ }^{2021}$ and inflamed ${ }^{22} 23$ colorectal mucosa. This variability in staining has been attributed to differing fixation and processing techniques and specificity and titre of the primary antibody. ${ }^{112021}$ Although fixation and processing techniques were standardised, we found a wide variation in carcinoembryonic antigen staining in this study. Carcinoembryonic antigen stained strongly in dysplastic and malignant tissues (both primary tumours and secondary deposits), although variable intensity was seen within some tumours. We could not consistently relate carcinoembryonic antigen staining to tumour differentiation. We can only agree with $\mathrm{O}^{\prime}$ Brien $e t a l^{21}$ that if a tumour fails to stain with carcinoembryonic antigen it is unlikely to be colorectal in origin. Furthermore, strong carcinoembryonic antigen staining is associated with colorectal carcinoma but is not by itself a reliable indicator of malignancy. It also appears to reflect epithelial proliferation, ${ }^{23}$ as in this context carcinoembryonic antigen presence in inflamed and regenerative mucosa prevented us from using it to distinguish from areas of dysplasia.$^{28}$ Future assessment using monoclonal antibody may give more well defined results.

The concept of the mucosal assembly of $\operatorname{IgA}-\mathrm{J}-$ secretory component ${ }^{12}$ was followed by reports from Poger $e t a^{24}$ and Weisz-Carrington $e t a^{25}$ using a frozen section immunofluorescent technique. They studied colorectal carcinomas and benign polyps and related diminution in secretory component to progressive epithelial dedifferentiation in both carcinoma and atypical areas in the adenomatous polyps. This has since been confirmed ${ }^{2627}$ and accords with findings in this study. Secretory component was present in five well differentiated tumours (three only focal staining), four of these being mucin secreting. Polypoid adenomatous foci showed light focal staining.

Several tumours showed stromal $\mathrm{IgA}$ and $\mathrm{J}$ chain staining but absent secretory component. This has been noted previously ${ }^{25}$ and may be related to impairment of production of secretory component or its transport through the epithelium. Presumably this functional impairment is incurred during the evolution of malignant change. In this context, Isaacson $^{27}$ noted in areas of villous dysplasia that some of the superficial epithelium had normal morphological appearances but lacked secretory component, which implies a functional dysmaturity. This has been found in the present material. Thus the absence of secretory component has emerged as a potential tissue criterion for malignant change. Two studies by Isaacson ${ }^{27}$ and Rognum et $a l^{28}$ have examined the effects of inflammation and dysplasia in ulcerative colitis on the tissue expression of secretory component. As in this study, they found more intense secretory component staining with differentiated tissue and lack of secretory component in dysplasia and carcinoma. In these instances the pathologist was usually in no doubt as to the absence or presence of dysplasia on routine haematoxylin and eosin assessment. It is in the borderline area of regenerative hyperplasia versus dysplasia that use of secretory component was suggested as a diagnostic aid. ${ }^{27}$ Isaacson found preservation of secretory component in regenerating mucosa and absence or reduction in dysplasia (with one exception). Rognum et al reported variable tissue antigen expression and concluded that secretory component could not be used as a discriminating factor in diagnostic work, secretory component staining being related to both the degree of dysplasia and inflammation. This study has shown more intense secretory component staining in inflamed and regenerative tissue than in dysplasia, but, also, multiple areas of similar regenerative morphology with reduction or absence of secretory component. We believe that this precludes at present the use of secretory component as a reliable diagnostic marker of early malignant change. This is particularly so when one considers that a decision for colectomy may be made on small mucosal biopsies rather than strips of mucosa.

The variation in secretory component expression in these studies is unlikely to be due to antisecretory component serum titre or specificity. ${ }^{27}$ The exact physiological relation between the production of free secretory component, its integration into assembled secretory $\operatorname{IgA}$, and the $\operatorname{IgA}$ containing mucosal plasma cells still remains to be fully established. ${ }^{25}{ }^{27}$ Assessment of secretory component as an isolated index of epithelial maturity, particularly in inflamed regenerative mucosa, is therefore doubtful.

In conclusion, this study used an immunoperoxidase technique for carcinoembryonic antigen, IgA, and $J$ chain in ulcerative colitis. The mucosal lesions of regenerative hyperplasia and indefinite and low grade dysplasia showed variable tissue antigen 
expression. This precluded the use of immunostaining as a reliable diagnostic aid in discriminating regenerative epithelial changes from true dysplasia. Secretory component warrants further study to elaborate its relation to mucosal plasma cells, inflammation, dysplasia, and epithelial expression.

We thank Miss Diane Barker, who kindly typed the manuscript.

\section{References}

' Crohn BB, Rosenberg H. The sigmoidoscopic picture of chronic colitis (non-specific). Am J Med Sci 1925;170:220-8.

${ }^{2}$ Warren S, Sommers SC. Pathogenesis of ulcerative colitis. Am J Pathol 1949; 25:657-79.

${ }^{3}$ Morson BC, Pang LSC. Rectal biopsy as an aid to cancer control in ulcerative colitis. Gut 1967;8:423-34.

4 Yardley JH, Keren DF. "Precancer" lesions in ulcerative colitis: a retrospective study of rectal biopsy and colectomy specimens. Cancer 1974;34:835-44.

${ }^{5}$ Riddell RH. The precarcinomatous phase of ulcerative colitis. In: Morson BC, ed. Current topics in pathology of the gastrointestinal tract. Berlin: Springer, 1976:179-219.

- Riddell RH, Goldman H, Ransohoff DF, et al. Dysplasia in inflammatory bowel disease: standardised classification with provisional clinical applications. Hum Pathol 1983; 14:931-68.

' Ehsanullah M, Filipe MI, Gazzard B. Mucin secretion in inflammatory bowel disease: correlation with disease activity and dysplasia. Gut 1982;23:485-9.

${ }^{8}$ Franzin G, Grigioni WF, Dina R, Scarpa A, Zamboni G. Mucin secretion and morphological changes of the mucosa in nonneoplastic diseases of the colon. Histopathology 1983; 7:70718.

${ }^{9}$ Lehy T, Mignon M, Abitbol JL. Epithelial cell proliferation in the rectal stump of patients with ileorectal anastomosis for ulcerative colitis. Gut 1983;24:1048-56.

${ }^{10}$ Hamarberg C, Slezak P, Tribukait B. Early detection of malignancy in ulcerative colitis: a flow-cytometric DNA study. Cancer 1984;53:291-5.

"Isaacson P. Tissue demonstration of carcinoembryonic antigen (CEA) in ulcerative colitis. Gut 1976; 17:561-7.

12 Poger ME, Lamm ME. Localisation of free and bound secretory component in human intestinal epithelial cells: a model for the assembly of secretory IgA. J Exp Med 1974;139:629-42.

${ }^{13}$ Isaacson P, Judd MA. Immunohistochemistry of carcinoembryonic antigen: characterisation of cross-reactions with other glyco-proteins. Gut 1977; 18:779-85.

14 Gold P, Freedman SO. Demonstration of tumour-specific antigens in human colonic carcinomata by immunological tolerance and absorption techniques. $J$ Exp Med 1965; 121:439-62. is Isaacson P, Le Vann HP. The demonstration of carcinoembryonic antigen in colorectal carcinoma and colonic polyps using an immunoperoxidase technique. Cancer 1976;38: 1348-56.

${ }^{16}$ Isaacson P, Judd MA. Immunohistochemistry of carcinoembryonic antigen in the small intestine. Cancer 1978;42: 1554-9.

"Sun NCJ, Edgington TD, Carpentier CL, McAfee W, Terry R, Bateman J. Immunohistochemical localisation of carcinoembryonic antigen (CEA), CEA-S and nonspecific cross-reacting antigen (NCA) in carcinomas of lung. Cancer 1983;52:163241.

${ }^{18}$ Albores-Saavedra J, Nadji M, Morales AR, Henson DE. Carcinoembryonic antigen in normal, preneoplastic and neoplastic gallbladder epithelium. Cancer 1983;52:1069-72.

${ }^{19}$ Bychkov V, Rothman M, Bardawil WA. Immunocytochemical localisation of carcinoembryonic antigen (CEA), alphafetoprotein (AFP) and human chorionic gonadotrophin (HCG) in cervical neoplasia. Am J Clin Pathol 1983;79:414 20.

${ }^{20}$ Primus FJ, Clark CA, Goldenberg DM. Immunoperoxidase localisation of carcinoembryonic antigen in normal human intestinal mucosa. J Natl Cancer Inst 1981;67:1031-9.

${ }^{21}$ O'Brien MJ, Zamcheck N, Burke B, Kirkham SE, Saravis CA, Gottlieb LS. Immunocytochemical localisation of carcinoembryonic antigen in benign and malignant colorectal tissues: assessment of diagnostic value. Am J Clin Pathol 1981;75:283-90.

22 Goldenberg DM, Sharkey RM, Primus FJ. Immunocytochemical detection of carcinoembryonic antigen in conventional histopathology specimens. Cancer 1978;42:1546-53.

${ }^{23}$ Wager C, Hain F, Breuer H, Olude S, Cremer H, Muller-Walraf R. Immunohistochemical demonstration of carcinoembryonic antigen in normal, transitional and inflamed colonic mucosa. Oncodev Biol Med 1981;2:331-43.

24 Poger ME, Hirsch BR, Lamm ME. Synthesis of secretory component by colonic neoplasms. Am J Pathol 1976;82:327-38.

${ }^{25}$ Weisz-Carrington P, Poger ME, Lamm ME. Secretory immunoglobulins in colonic neoplasms. Am J Pathol 1976;85:303-16.

${ }^{26}$ Rognum TO, Elgjo K, Brandtzaeg P, Ørjasaeter H, Bergan A. Plasma carcinoembryonic antigen concentrations and immuno-histochemical patterns of epithelial marker antigens in patients with large bowel carcinoma. J Clin Pathol 1982;35: 922-33.

${ }^{27}$ Isaacson P. Immunoperoxidase study of the secretory immunoglobulin system in colonic neoplasia. J Clin Pathol 1982;34:14-25.

${ }^{28}$ Rognum TO, Elgjo K, Fansa O, Brandtzaeg P. Immunohistochemical evaluation of carcinoembryonic antigen, secretory component, and epithelial IgA in ulcerative colitis with dysplasia. Gut 1982;23:123-33.

Requests for reprints to: Dr Derek Allen, Histopathology Laboratory, Belfast City Hospital, Belfast BT9 7AD, Northern Ireland. 